\title{
Acoustic Emission (AE) as a diagnostic tool in geophysics
}

\author{
Gabriele Paparo $\left({ }^{1}\right)$, Giovanni P. Gregori $\left({ }^{1}\right)$, Ugo Coppa $\left({ }^{2}\right)$, Riccardo de Ritis $\left({ }^{1}\right)$ \\ and Alberto Taloni $\left({ }^{1}\right)$ \\ (1) Istituto di Acustica (IDAC-CNR), Roma, Italy \\ (2) Osservatorio Vesuviano, Napoli, Italy
}

\begin{abstract}
Acoustic Emissions (AE) are effective for monitoring ground deformation and temporal variation of its porosity. $\mathrm{AE}$ are complementary to seismic information, related to the same area, though $\mathrm{AE}$ and earthquakes focus on observational evidence concerned with substantially different space- and time-scales. AE information is pertinent (i) either for geodynamically stable areas, where it probes the diurnal thermal and/or tidal deformation, (ii) or for seismic areas where it provides some as yet unexploited precursors, (iii) or for volcanic areas, where it appears capable of recognising precursors originated by some hot fluid that penetrates by diffusion into rock pores, from those associated with eventual plutonic magma intrusions, (iv) and also for monitoring periods of time during which a volcano is «inflated» by underground hot fluids compared to others during which it «deflates». Upon direct comparison between 6 data sets concerned with different physical settings, it seems to be possible (fig. 3 and table II) to distinguish a few significantly different behaviours associated either (i) with a mere compression (such as occurs for Stromboli, Vesuvius, and a sample compressed in the laboratory), or (ii) with a slip strain, such as it typically occurs in association with faulting or with diurnal thermal rock deformation.
\end{abstract}

Key words acoustic emission - precursors earthquakes - volcanoes - dilatancy

\section{Introduction}

We address the heuristic potential of Acoustic Emissions (AE) as a diagnostic tool for studying microscale processes associated with ground deformation, microfracturing, and porosity variation. Such application can be concerned (i) with the study of matter exchanges across soil surface, or (ii) with a few different processes that occur within the ground and that are associated

Mailing address: Ing. Gabriele Paparo, Istituto di Acustica (IDAC-CNR), Via Fosso del Cavaliere 100, 00133 Roma, Italy; e-mail: paparo@idac.rm.cnr.it either with thermal variations, or with tectonic activity, or with hydrothermal or volcanic phenomena. AE applications are normally almost neglected in geophysics, depending on intrinsic difficulties in AE propagation through disjointed ground, and on their random detection capability. Such previous standard defeatist feeling can be overcome considering AE like a yelp, whine, moan or whimper, ... that we can only occasionally catch. You may eventually be capable of hearing it or missing it, whether loud or feeble. However, whenever you detect it, you should afford to recognise its meaning. Hence, the focus is on seldom-detected AE messages by the system. Observations prove how, in several circumstances, some very useful AE signals are detected, which provide relevant unprecedented information. Our purpose is to show the concrete feasibility of such applications, including 
comparison of field data with AE released by laboratory samples. A few examples referring to different tectonic settings and their potential are discussed, including hints for further developments.

The key relies on methodology. We make a simple application of fractal algorithms. The analysis is not concerned with the AE amount, frequency, intensity, or energy, etc. AE are released by an unknown widespread source distribution, and several sources, e.g., of anthropic origin, have no real physical relation with the effect of concern.

Our method, when considered a posteriori, is very robust with respect to anthropic or natural perturbations. However, it would be presumptuous (either in the negative or in the positive sense) to compare either our records, methods of analysis, or results, with previous investigations. There is no «better» or «worse» method. A given database and/or method of analysis can be suited or not for some given purpose. Different approaches must be realistically compared with each other with no preconceived understatement or overstatement.

\section{The data}

Table I summarises our data set. Two frequency ranges were monitored. The time sequence of $\mathrm{AE}$ events at different frequencies appears correlated and time-shifted with respect to each other. Every acoustic transducer (e.g., Cuomo et al., 2000) gives a signal in terms of an electric potential. In general, unless otherwise stated, for geophysical applications the transducer signal was recorded averaged over $\sim 25 \mathrm{~s}$, while in laboratory experiments no averaging was applied.

The following definitions were used: i) An AE burst is called a raw record, with some high time resolution, typically similar e.g., to a seismogram, though on different scale. ii) An AE microevent is a record of the sum of the maximum amplitudes of all bursts occurring during every time interval of $\sim 10 \mathrm{~ms}$. A microevent is the sum of several bursts. iii) An $\mathrm{AE}$ event is similar to a microevent, though in terms of a much longer averaging time interval. An event is the sum of several microevents. The typical time scale required for defining an event depends on the physical system. For instance,

Table I. The AE data base.

\begin{tabular}{lcl}
\hline \hline Site & $\begin{array}{c}\text { Monitored } \\
\text { frequency }\end{array}$ & \multicolumn{1}{c}{ Comments } \\
\hline $\begin{array}{l}\text { Gran Sasso } \\
\text { Southern Apennines (Potenza) }\end{array}$ & $\begin{array}{c}200 \mathrm{kHz} \\
25 \mathrm{kHz}\end{array}$ & $\begin{array}{l}\sim 4 \text { years records available (fig. 1). } \\
\text { Several records available, including one case history } \\
\text { on the occasion of a } M_{d}=4.6 \text { earthquake with epicentre }\end{array}$ \\
& $200 \mathrm{kHz}$ & $\sim 18.2 \mathrm{~km}$ from the AE recorder (fig. 4b). \\
\hline Stromboli & $160 \mathrm{kHz}$ & $\begin{array}{l}\text { A few years of discontinuous data available, with } \\
\text { techniques slightly improved versus time, still working. }\end{array}$ \\
& $25 \mathrm{kHz}$ & $\begin{array}{l}\text { Several discontinuous data monitored since 1995, and } \\
\text { also recently after October 1999 by means of an }\end{array}$ \\
& $200 \mathrm{kHz}$ & $\begin{array}{l}\text { improved instrumentation during a crisis period; the } \\
\text { recording site is located almost on the top of the } \\
\text { velcano, very close to the rim of its crater, still working. }\end{array}$ \\
& $\begin{array}{l}\text { A cylindrical unconfined sample was compressed } 6 \\
\text { times, by a force transversal to its axis, although not } \\
\text { implying the final destruction of the sample (fig. 2). }\end{array}$ \\
\hline Laboratory sample & $200 \mathrm{kHz}$ &
\end{tabular}




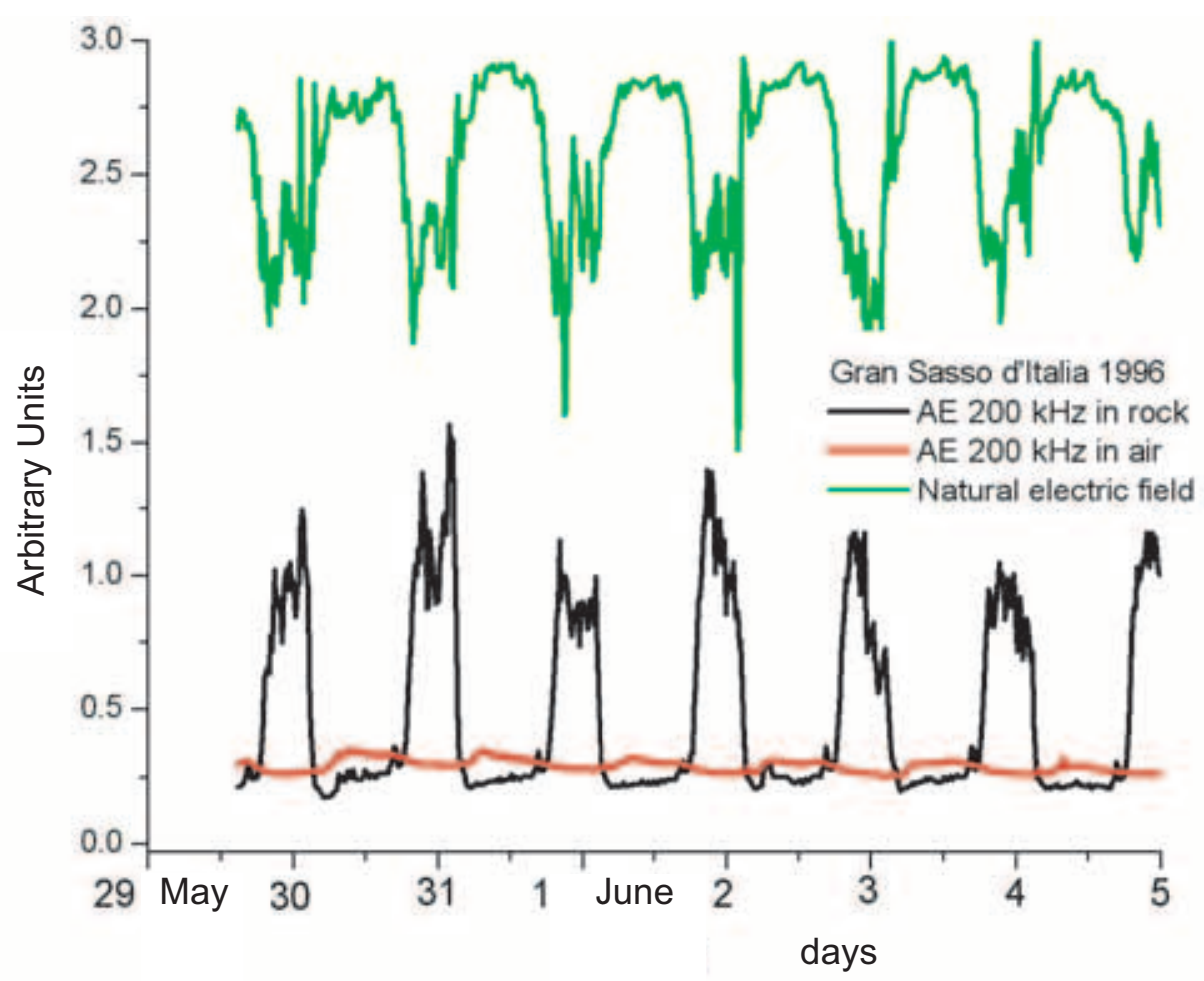

Fig. 1. Records in the Gran Sasso area, May 29 through June 5, 1996, showing AE at $200 \mathrm{kHz}$ both inside rock (lower black) and in air (blank signal, lower light grey) and the natural electric field (upper plot) recorded by metal rod inserted into ground for $\sim 12 \mathrm{~m}$ (in co-operation with INGV) and used as a receiving antenna tuned on the same frequency.

an event within a stressed alloy implies phenomena that occur to the micro-crystal structure of the sample within short time scales. A phenomenon occurring within a tectonic setting relies on some prime yielding bonds that are other than within a sample stressed in the laboratory. We characterised an event by means of an amplitude, which is the average amplitude of all microevents falling inside some given pre-chosen acquisition time-lag (for every geophysical application, we currently chose $\sim 25 \mathrm{~s}$ ). Such AE time series was suitable for investigating geophysical settings.

Gran Sasso is the main massif (composed mostly of dolomia and limestone) of the Apennines in Central Italy. On the time scale of our records, its tectonic setting is comparatively stable, notwithstanding its surrounding area is prone to seldom occurring strong earthquakes, although with much longer return-times. The records (fig. 1) resulted significant, displaying some very regular daily variation, its day to day difference being likely related to climate (see below). The AE transducers were put both on top of a steel bar inserted into the rocky ground for $\sim 12 \mathrm{~m}$, while another was hanging in the air (blank signal). The natural electric field was recorded (in co-operation with INGV) using the same steel bar as a receiving antenna tuned on the same frequency. The AE are conspicuous during the daily cooling of ground (local nighttime). The diurnal cooling of rocks makes their external layer contract over a warmer and temporarily more expanded interior: cleavage 
rupture must occur. The record of the natural electric field is associated with the ionospheric tide (contrary to oceanic and solid Earth's tides, the atmospheric tides are well known to be mostly thermal and only at a much lesser extent also gravitational). Since Marconi's times, such (eventual) expected natural AE disturbance is well known to display a violent maximum during daytime. In contrast, in our experiment, both $\mathrm{AE}$ signals (the one actually recorded, and the one expected being originated by rock cooling) show a maximum during nighttime. That is, for sure there is clear anticorrelation between ionospheric disturbances and the recorded AE signal. Hence, no ionospheric effect enters into the AE records. Moreover, the blank signal monitors eventual disturbances originated from any kind of external sources (either anthropic, or ionospheric, or other, i.e. generated by sources that in no case can be related to underground phenomena, or within the electronics of the recording system, etc.). Such disturbances, if they exist, should cause the same effect both on the sensor within air and on the sensor put on the metal rod. Our results very clearly show that no such perturbation affects our AE records (at least as far as our Gran Sasso monitoring is concerned). Therefore, at least at middle latitudes and in the absence of major anthropic disturbances, one can safely put AE transducers either on an insulator bar, or on a metal rod, with no bias on measurement. Under such circumstances, every opposite conclusion (e.g., such as Diodati et al., 2001; a formal comment to that paper submitted to that journal was not published; no reply was given to our objections) appears mere nonsense.

The Southern Apennines site (Giuliano) is close to Potenza, within a tectonically active area that during the time spanned by our records suffered an $M_{d}=4.6$ earthquake. The two AE transducers (for two frequencies) were put on top of two steel bars inserted into a solid flysch outcrop.

Stromboli is a volcanic island with an unclear tectonic nature. Its isotopic chemism appears more reminiscent of an oceanic island rather than of an island arc (Crisci et al., 1991; Esperança et al., 1992; Esperança and Crisci, 1995). For over $\sim 3$ millennia Stromboli has made $\sim 100$ explosions per day, resulting in an unprecedented natural environmental laboratory (e.g., Chiappini et al., 2002; Gregori et al., 2001; Paparo and Gregori, 2001). Its volcanic edifice is comparable in size to Etna's, though almost completely immersed within the sea. The AE transducers were located on top of two $\sim 1 \mathrm{~m}$ bars (one made of steel, one of glass) inserted into a rock dyke.

Vesuvius is (with Etna) the historically bestdocumented volcano. It had its last effusive eruption in 1944. Since that time it has been apparently quiescent, although its seismic activity recurrently and irregularly experiences disquieting periods. Its historical eruption series display some regularities (Gregori et al., 1992; Gregori, 1993, 1996), apparently supported by a correlation with the activity versus time of every other historical volcano of the world (Gregori et al., 1994). AE monitoring may provide some unprecedented information on new degrees of freedom for diagnosing the state of the system. AE transducers were located on top of $\sim 1 \mathrm{~m}$ glass bars inserted into a lava dyke, outcropping almost on the very top of the volcano, at an axial distance of a few hundred metres from its crater.

Gran Sasso can be likened to some unique rocky block. At all other sites, AE propagation can be biased by damping while crossing disjointed material. On the other hand, the collected observations guarantee per se as a matter of mere observational fact about such concern. On a speculative basis, three possible ways can be envisaged.

First, one can presume that some solid rocks, almost like elongated ores crossing through otherwise disjointed soil, are capable of acting like wave-guides for AE propagation. In principle, such possibility can be checked by some dense array of AE detectors: spatial distribution, crossed correlation, and time shift of $\mathrm{AE}$ records within the array should disclose such speculated wave-guides. AE should appear to be a local phenomenon, implying a serious drawback due to the choice of the critical location of the AE transducers.

Second, speculate that some teleconnection mechanism at much Lower Frequencies (LF) than AE's makes the signal to propagate through disjointed material. Whenever it stresses some rock, it triggers High Frequency (HF) AE. In such case, whenever AE records are carried out within 
some suitable array and within some wide fan of different frequencies, the LF propagation should be directly detected, including the time- and spaceseries of the HF AE trigger. Detector location is critical, although AE should be almost ubiquitous and correlated with local geology.

Third (and more likely), some entire area, or physical volume, on the regional or maybe even continental scale is deformed, notwithstanding no effect is detected by any standard method. Some strain, however, is in progress over the entire area, and it occurs basically everywhere. At some very small local scale, i.e. wherever some geological solid formation is present, the $\mathrm{AE}$ are monitored by transducers put right on top of such bodies. The AE results are a strictly local phenomenon (detected only whenever the transducer is put right on top of a suitable solid body). They result from the strain of some very local solid feature, although being the consequence of some large-scale stress. That is, $\mathrm{AE}$ monitors a yelp, whine, moan or whimper, ... of some comparatively very small solid body, which is part of some huge geological region that is suffering from some large-scale deformation. Differently stated,

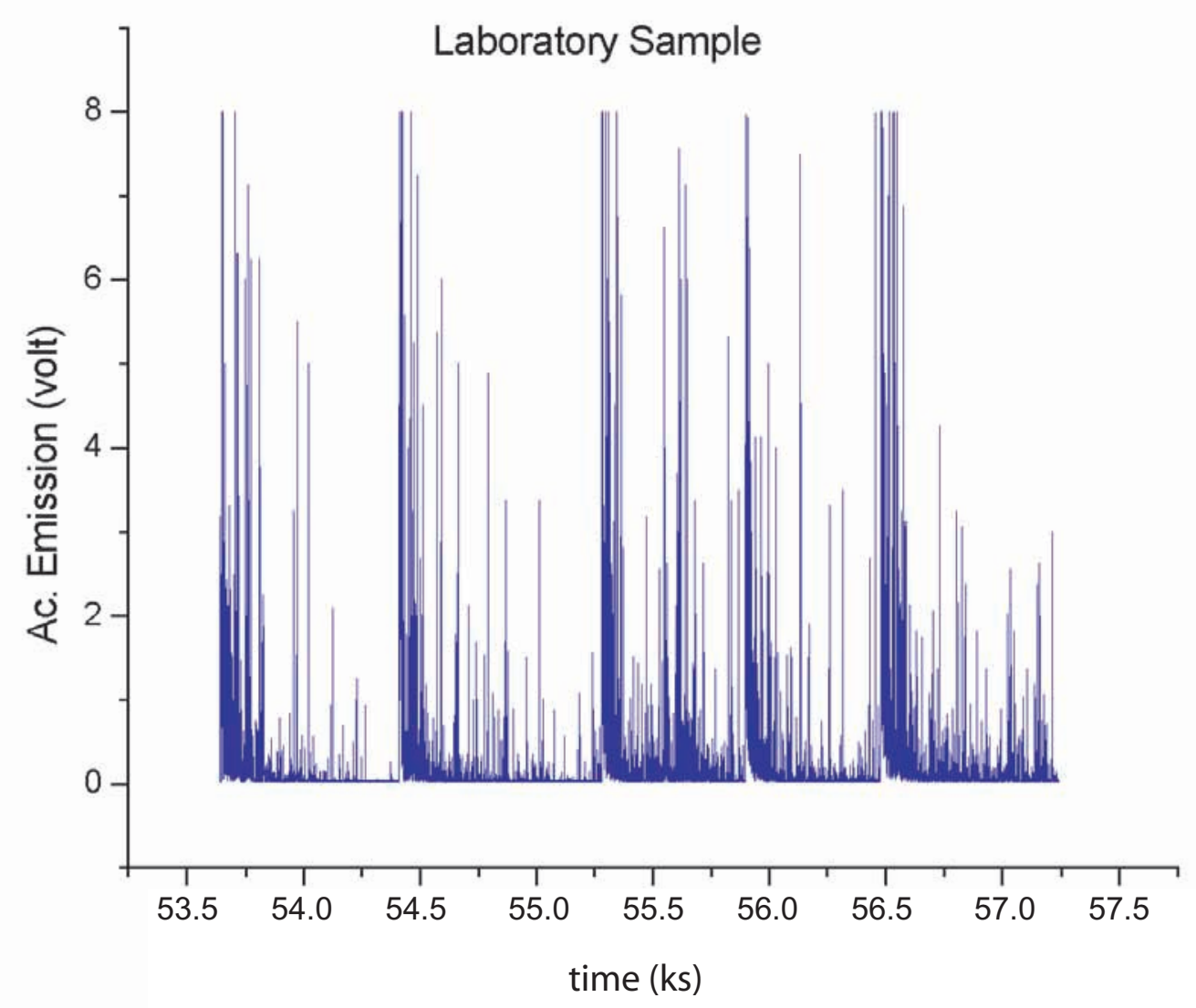

Fig. 2. AE bursts recorded during a laboratory experiment. A solid sample composed of a mix of Gran Sasso limestone was compressed and decompressed. The five stronger AE sequences correspond to increased stress. The highest peaks denote amplifier saturation level. The sampling rate is $200 \mathrm{~ms}$. Redrawn after Petri et al. (1994) and Vespignani et al. (1995). 
the monitoring apparatus contains, as an essential constituent, also the solid body that extends deep into the ground to some unknown range. Such solid body is like a true natural tentacle probing some fraction of a large geological and geophysical system, and capable of monitoring (just as a mere matter of observational fact) its reaction to stress and strain.

The second and third such possibilities are ultimately the same, their difference being only mathematical. The second possibility refers to some teleconnection by means of one given LF wave, while the third possibility refers to some large scale deformation, the «signal» of which can be mathematically Fourier decomposed into a continuous spectrum of LF waves.

On a mere intuitive basis, the first and second ways appear less realistic than the third one. It does not seem possible to envisage any different possible way. Hence, the third possibility appears more likely.

No array of AE transducers was available to the authors. In every case history, however, AE signals definitely seem to be physically significant and useful for geophysical monitoring. Their real heuristic potential is presumably different in different applications, depending on tectonic setting, local morphology and eventual disturbances, etc. In addition, some AE records were also considered for comparison purposes, collected in the laboratory while stressing and straining a solid sample obtained from a mix of Gran Sasso limestone (fig. 2).

Seismic data are complementary, suitable for correlation with AE. They reflect a later stage of the evolution of the system. Observations seem consistent with the hypothesis that phenomena always start by HF AE ( 160-200 kHz) (originated when some very small pores yield), followed by LFAE $(\sim 25 \mathrm{kHz})$ (when pores yield and coalesce into larger microcavities), and finally at some later time by an earthquake $(\sim 1 \mathrm{~Hz}$, when the large scale mechanical structure of the system yields). Hence, in principle, one should expect that a hypothetical monitoring carried out by some large set of different frequencies should detect a clear sequence of AE signals of progressively decreasing frequency. Such sequence, however, ought to span a limited frequency range, because, according to the aforementioned third explanation, $\mathrm{AE}$ is concerned with local geological structures of limited spatial extension, unlike the large seismic energy release that ought to be originated by a tectonic structure other than the AE source, though both sources respond to a common prime deformation and trigger. Concerning Vesuvius, a careful seismic log has been available since 1972 A.D. Seismic data for the Potenza area, in Lucania, Southern Apennines, were available from INGV, although spanning some limited period.

\section{The physics}

Ground deformation is originated by either one of the following prime physical causes: i) thermal contraction and expansion; ii) tidal fluctuation; iii) tectonic strain; iv) endogenous pressure by a fluid (i.e. water, oil, geogas, etc.); and $v$ ) endogenous pressure by plutonic intrusion of magma. Every such process implies phenomena that have to be studied beginning from their atomic or molecular scale through their respective large-scale effects. AE are triggered whenever some chemical bond yields. The phenomenon eventually propagates like a chainreaction, eventually leading to strain deformation or to rupture, with morphology, timing, and speed, which depend altogether on the structural environment and on the prime trigger. A mere compression e.g., by a hot fluid that diffuses into the pores can be expected to imply a different behaviour than the case of a lateral displacement, such as occurs for crystal cleavage or tectonic faulting. Every such feature must be physically fitted. The temporal evolution of AE series closely depends on the prime energy source, hence on the morphology and geometry of the stress and subsequent strain.

This recalls the classical dilatancy hypothesis (e.g., Nur, 1972; Rikitake, 1976; Mogi, 1985) for explaining earthquakes precursors. According to the rationale of the present study, the model here speculated ought to be considered as a peculiar case of some wider and more general conceptual perspective. Much as always occurs in every discipline, every understanding is based on some simplifying abstraction. Therefore, its consequent model, aimed at explaining some specific ob- 
servations in some detail, is eventually physically significant only, and strictly only, within the limits of its own basic approximations, and never outside them.

Thermal (non-endogenous) effects are originated by a source external to the rock. This occurs in the case of solar diurnal heating and cooling that penetrates only down to several tens of centimetres. Whenever such warming reaches some depth, the rock layer can freely expand and the process is smooth. In contrast, whenever a rock layer cools off, its outer layers contract while they are compelled to contain internal layers that are still more expanded. Hence, a cooling (unlike a warming) object ought thus to imply some lateral displacement (i.e. it is a 2D slip of the shrinking outer layers). Such phenomenon occurs within some shallow layer, and it should occur only on the surface, unless there are some peculiar structures that act like the aforementioned AE wave-guides to deeper layers.

Tidal deformation uniformly applies to the entire Earth. Compared to thermal deformation it appears likely to be a smooth phenomenon, originated in $3 \mathrm{D}$ space that should emit some lesser AE, or even no AE at all. For instance, every huge building does not necessarily release $\mathrm{AE}$ or crunching, notwithstanding it actually experiences conspicuous diurnal tidal oscillations (typically by several tens of centimetres).

Tectonic strain should imply AE mainly originated by lateral displacement (here called tout court «slip» AE), much as occurs for thermal deformation, though occurring over some much wider surface, i.e. on the slip plane of a fault, which can be either of small or large extension. The analogy between slip AE associated with either thermal or tectonic strain seems to be almost perfect, the difference relying on the fact that the thermal case is concerned with ruptures of atomic bonds within a crystal structure, while tectonic strain is concerned with cohesion forces within the ground, which characterise soil hardness and that under different settings avoid land slides. Such bonds are concerned with soil physics (unlike e.g., in an alloy, or in the concrete of a building, where the yielding bonds refer to rupture along micro-crystal cleavage planes). A still different physical environment is concerned with snow-slides, i.e. with snow or ice hardness, provided that it is possible to perform some $\mathrm{AE}$ monitoring at the appropriate location. In such case history, the AE are released depending on ice physics. It ought to be emphasised, however, that the slip AE observed on the occasion of a tectonic strain could be either originated over the fault plane, or also by the microfracturing of the rocky body over which the transducer was located. The mathematical rationale and method of analysis may eventually be the same, though applied to physically different systems and case histories.

The endogenous pressure by a fluid ought to be a typical feature of every system dominated by hydrothermal or phreatic breeding. AE sources should be distributed into a $3 \mathrm{D}$ region, where $3 \mathrm{D}$ fluid diffusion occurs, and therefore they should display their own signature, distinct from AE originated by an externally imposed stress, which should recall some more organised, less 3D and comparatively more $2 \mathrm{D}$, spatial distribution.

The endogenous pressure by plutonic intrusion of magma has to be likened to a tectonic strain. In fact, fluids such as water, oil, geogas, etc. compared to magma have a very great mobility and compressibility. Therefore, they can easily reach the smallest pores, and exert, almost randomly, a pressure that rapidly propagates by 3D diffusion, producing local micro-cracks, and coalescence into progressively larger microcavities, etc. In contrast, a plutonic body has some extremely low mobility, and produces only very slow ground deformation, often undetectable from the ground surface, at least until the phenomenon is not in some comparatively late stage of evolution. Such effects are generally reported by standard observations during the few days preceding the opening of a new boca (implying ground upheaval by several tens centimetres). The two case histories are therefore very different. In the case that a plutonic intrusion is also associated with hydrothermal or gas propagation underground, AE will detect both such kinds of event. Hence, the leading (though not the unique) effect of a plutonic intrusion, prior to its large-scale late manifestation as ground deformation, ought to be likened to some comparatively more or less large or small tectonic deformation, implying 2D slip along a surface of some small (and undetectable from 
surface) faults. In every such case this is more a 2D than a 3D phenomenon. It should be stressed that in every such case history, the comparatively more $2 \mathrm{D}$ than $3 \mathrm{D}$ character of the recorded $\mathrm{AE}$ is not necessarily associated with some large 2D slip surface or fault plane. Since we are interested in the character of the prime temporal sequence of $\mathrm{AE}$ associated with a reaction chain while the chemical bonds yield, even phenomena occurring along the slip plane of sub-microscopic crystal size do produce the $2 \mathrm{D}$ expected effect, even when no «macroscopic» slip or fault plane etc. can be detected.

One very similar occurrence, though in the space (linear) scale of $\sim 10000 \mathrm{~km}$ and on the geologic time scale, is concerned with superswells (typically observed in the Pacific area where seamounts are a dense array of natural mareographs). According to the evidence discussed by Gregori and Dong (1996) and based on the output of the Hawaii hot spot, the push by the endogenous thermal source appears to be the origin of some generalised large-scale uplift, i.e. the superswell, by which plates start sliding by gravity on its slopes, thus acquiring kinetic energy. Such stage often precedes the outburst of a Large Igneous Province (LIP). Whenever this occurred on emerged land, even the river drainage recorded such large-scale slopes (Cox, 1989). The kinetic energy is later transformed into friction heat, and by $\sim 50000-100000$ years it is finally manifested as a large increase in volcanism.

Consider a laboratory experiment and compress a rock sample, while it is kept laterally confined. It is expected that the AE be similar to the case of an endogenous 3D pressure alone, exerted e.g., by a fluid. However, if the sample is not confined, a smaller percentage of transversal slip occurs and its associated 2D slip AE.

Summarising, the key-item of such entire discussion deals with the distinction between 2D and $3 \mathrm{D}$ distribution of the prime $\mathrm{AE}$ sources. It is physically characterised in terms of a different timing between subsequent yields of the bonds (either within crystals, or within ground). This is the rationale for recognising the kind of yelp, whine, moan, whimper, .... The theory of stress and strain of a non-perfectly elastic material was extensively investigated for purposes other than geophysics (e.g., Scott, 1991). Moreover, as far as an explicit calculation is concerned of the associated formal Schrödinger equation, it is a most difficult job even for the computation of a pure crystal, while it is essentially impossible for an alloy or for every unknown texture of different substances such as occurs in natural rocks or ground (see e.g., Eberhart, 1999).

AE's are complementary, from the viewpoint of the aforementioned dilatancy hypothesis, to other standard methods (topographic/bathymetric or seismic or GPS techniques, or repeated geodetic prospecting, tiltmetry and strainmetry, high sensitivity gravimetry, etc.) in the fact that they reflect the stage of the micropore yielding occurring on strictly local and very partial geological structures, long before the much larger mechanical deformation and final rupture that causes an earthquake. In any case history, every natural system is to be expected to be largely heterogeneous. Hence, AE ultimately monitor some structure of limited size, unless it responds to some large-scale teleconnection. In either case, however, it is clearly indicative of some wide and basically unknown, although never random, process of the system, originated at some more or less great depth, and involving some extended volume, or area. Owing to this fact, the AE information that can be monitored on the ground surface ought to be better conceived in terms of a monitoring array, rather than as a point-like record.

Considering the present state-of-the-art outlined in Section 1, the main purpose of the present study is a feasibility assessment about the effective heuristic potential of the $\mathrm{AE}$ technique. Moreover, the analysis here envisaged is focused on time series of AE events, while the possibility of inferring some analogous and more precise information directly derived from the temporal evolution of every single $\mathrm{AE}$ «event» is currently in progress.

\section{The analysis}

\subsection{Fractal properties}

Consider the timing of the AE events, and infer whether their sources are 2D or 3D distributed. Neglect the intensity of every AE 

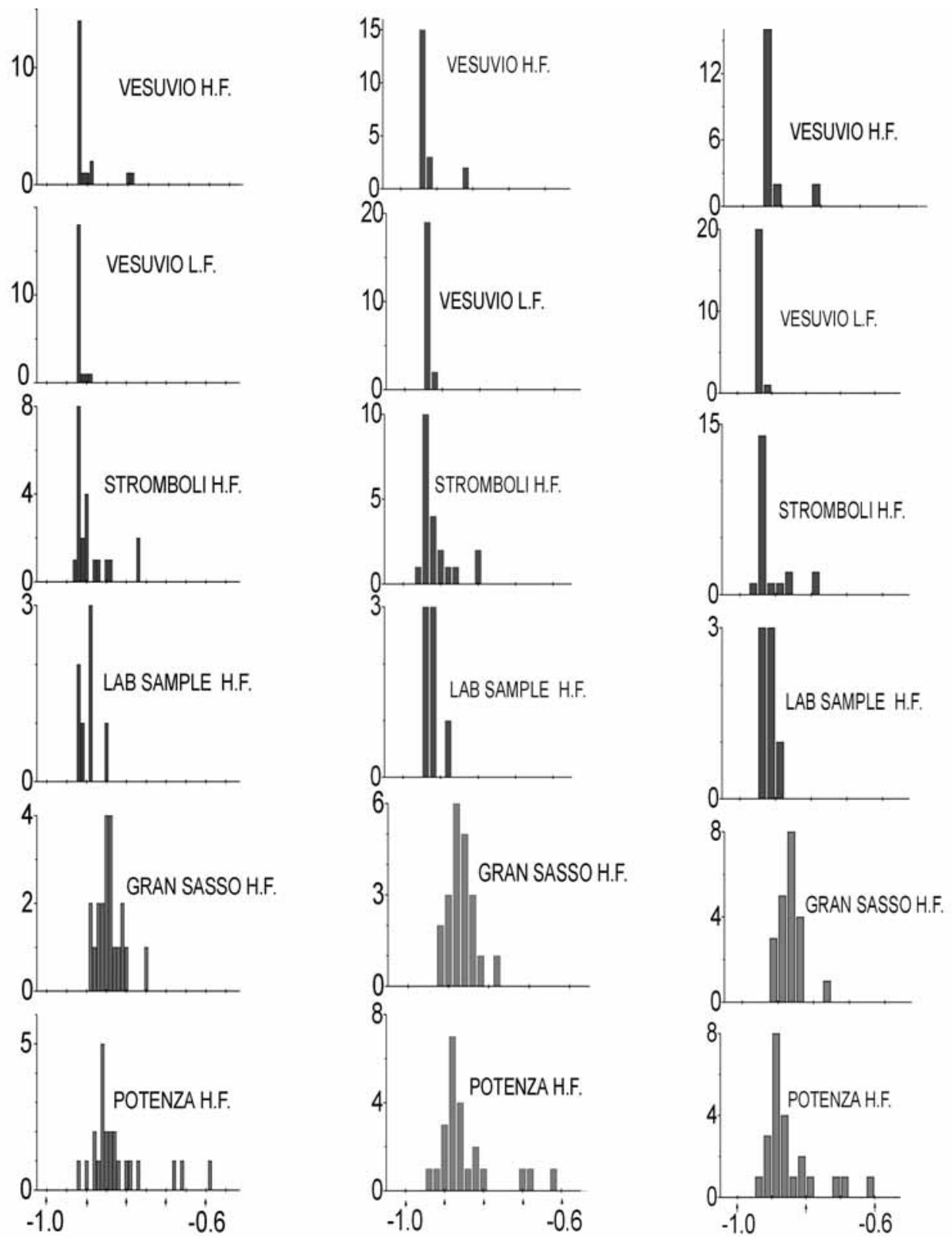

Fig. 3. Statistical distributions of the slopes $H$ computed by the box counting method applied to the 6 case histories listed in table I. The observed distributions seem to partake into two families with $D \sim 1$. and $D \sim 0.9$, respectively. Three different histograms for every case are plotted, being defined by the identical data set, though by using different elementary intervals on abscissas. The significance of distinguishing such distributions is tested by means of standard algorithms as per table II. The lowest two lines of histograms are shown with a lighter shading in order to emphasize that their peaks occurr at $D \sim 0.9$, unlike others that are peaked at $D \sim 1.0$ 
Table II. Similarity of the fractal dimensions $D$ of the AE event sequences observed in different environments and case histories.

\begin{tabular}{|c|c|c|c|c|c|c|}
\hline & $\begin{array}{l}\text { Vesuvius } \\
200 \mathrm{kHz}\end{array}$ & $\begin{array}{l}\text { Vesuvius } \\
25 \mathrm{kHz}\end{array}$ & $\begin{array}{c}\text { Stromboli } \\
160 \mathrm{kHz}\end{array}$ & $\begin{array}{l}\text { Laboratory sample } \\
200 \mathrm{kHz}\end{array}$ & $\begin{array}{c}\text { Gran Sasso } \\
200 \mathrm{kHz}\end{array}$ & $\begin{array}{r}\text { Potenza } \\
25 \mathrm{kHz}\end{array}$ \\
\hline Vesuvius & & 0.079 & 0.33 & 0.70 & 0.000002 & 0.0001 \\
\hline $200 \mathrm{kHz}$ & & 0.085 & 0.33 & 0.62 & 0.000002 & 0.0001 \\
\hline 0.97929 & & $1.710^{-8}$ & 0.39 & -- & 0.56 & 0.0016 \\
\hline \pm 0.00015 & & 1. & 1. & 0.22 & 1. & --- \\
\hline 0.98784 & & 0.17 & 0.36 & --- & 0.000002 & 0.0000008 \\
\hline \pm 0.00003 & & & & 0.036 & & \\
\hline Vesuvius & 0.079 & & 0.009 & 0.0014 & $1.210^{-12}$ & 0.0000017 \\
\hline $25 \mathrm{kHz}$ & 0.085 & & 0.012 & 0.0491 & $5.510^{-10}$ & 0.0000065 \\
\hline 0.99485 & $1.710^{-8}$ & & $4.8 \cdot 10^{-10}$ & 0.0017 & $3.710^{-7}$ & $1.210^{-14}$ \\
\hline \pm 0.000009 & 1. & & 1. & --- & 1. & --- \\
\hline 0.99588 & 0.17 & & 0.006 & 0.0021 & $1.110^{-8}$ & $2.210^{-8}$ \\
\hline \multicolumn{7}{|l|}{ \pm 0.00001} \\
\hline Stromboli & 0.33 & 0.009 & & 0.72 & 0.0004 & 0.0012 \\
\hline $160 \mathrm{kHz}$ & 0.33 & 0.012 & & 0.62 & 0.0004 & 0.0011 \\
\hline 0.9664 & 0.39 & $4.8 .10^{-10}$ & & 0.091 & 0.15 & 0.0185 \\
\hline \pm 0.0002 & 1. & 1. & & --- & 1. & --- \\
\hline 0.8455 & 0.36 & 0.006 & & 0.43 & 0.00004 & 0.00009 \\
\hline \multicolumn{7}{|l|}{ \pm---} \\
\hline Laboratory & 0.70 & 0.0014 & 0.72 & & 0.0004 & 0.0202 \\
\hline sample & 0.62 & 0.0491 & 0.62 & & 0.0002 & 0.0004 \\
\hline $200 \mathrm{kHz}$ & --- & 0.0017 & 0.091 & & 0.366 & 0.0055 \\
\hline 0.97320 & 0.22 & --- & --- & & --- & --- \\
\hline \pm 0.00005 & --- & 0.0021 & 0.43 & & 0.0021 & 0.0034 \\
\hline \multicolumn{7}{|l|}{0.97297} \\
\hline \multicolumn{7}{|l|}{ \pm 0.00007} \\
\hline Gran Sasso & 0.000002 & $1.210^{-12}$ & 0.0004 & 0.0004 & & 0.28 \\
\hline $200 \mathrm{kHz}$ & 0.000002 & $5.510^{-10}$ & 0.0004 & 0.0002 & & 0.27 \\
\hline 0.9176 & 0.56 & $3.710^{-7}$ & 0.15 & 0.366 & & 0.00025 \\
\hline \pm 0.0001 & 1. & 1. & 1. & --- & & --- \\
\hline 0.9121 & 0.000002 & $1.110^{-8}$ & 0.00004 & 0.0021 & & 0.70 \\
\hline \multicolumn{7}{|l|}{ \pm---} \\
\hline Potenza & 0.0001 & 0.0000017 & 0.0012 & 0.0202 & 0.28 & \\
\hline $25 \mathrm{kHz}$ & 0.0001 & 0.0000065 & 0.0011 & 0.0004 & 0.27 & \\
\hline 0.8970 & 0.0016 & $1.210^{-14}$ & 0.0185 & 0.0055 & 0.00025 & \\
\hline \pm 0.0006 & --- & --- & --- & --- & --- & \\
\hline 0.8452 & 0.0000008 & $2.210^{-8}$ & 0.00009 & 0.0034 & 0.70 & \\
\hline \pm --- & & & & & & \\
\hline
\end{tabular}


event. As a first-trial, apply the box counting method. We considered 21 case histories for every one of the 6 different data sets listed in table I (we used 23 samples of Southern Apennines records, while only 6 data sets were available for the laboratory experiment). Concerning Vesuvius and the Southern Apennines records, also the time variation of the fractal properties were investigated.

The box counting method was applied as usual, by least-square fitting on the plotted points in the log-log diagram (Richardson's plot) of number of counts versus sampling interval $\mu$, and after rejecting the ruler intervals biased by limited statistics. The fractal behaviour, however, is missed whenever $\mu$ is excessively short, i.e. when it is comparable either with the minimum time resolution of the recorder or, with the shortest time interval elapsing between two consecutive bond yields. Similarly, when $\mu$ is excessively long compared with the total time lag spanned by the AE records, large statistical fluctuations enter into play. Hence, the fit must be limited within a suitable interval $\mu_{1}<\mu<\mu_{2}$. In every application the numbers of fitted points were always large and comparable with each other. The (negative) slope $H$ of the line in the Richardson plot is the fractal dimension $D=-H$. Refer to Turcotte (1992, eqs. (2.1) and (7.12)) for whom a fractal is defined whenever the number $N_{n}$ of objects or fragments composing a system associated with a characteristic linear dimension $r_{n}$ is $N_{n}=C / r_{n}{ }^{D}$ where $r_{n}=L / n$ and $C$ is a constant, $D$ is the fractal dimension, and $L$ is the total span of the database over which the box counting method is applied.

Hence, comparatively smaller slopes $H$ ought to be displayed by slip $A E$ (which are likely to be comparatively more $2 \mathrm{D}$ than $3 \mathrm{D}$ ) compared to compression AE (which have a 3D space distribution). A uniform time-distribution of the events ought to imply $D=1$. Moreover, it can also be formally shown that it must always be $0 . \leq D \leq 1$. (Gregori, 1998). Just by a matter of definition, every truly random time series of $\mathrm{AE}$ events has the same $D$ as a uniform AE series. Therefore, every kind of law that makes the observed AE time series deviate from such ideal and mere randomness shall imply some corresponding reduction of $D$. Hence, if the box counting method gives $D=1$. we infer that the

\section{Comments to table II}

The result of every respective comparison is here denoted by the probability that every two given observed distributions have either a similar or a significantly different value of some given parameter, or that they be the same or not. For instance, a very low probability means that it is very likely that the two given distributions are significantly different compared with each other. It is customary to consider e.g., a threshold of 0.05 , by which, when the probability is $<0.05$ the two distributions are considered significantly different. Different printing characters are used to give direct visual evidence of a few different ranges of such probabilities. Large bold characters denote distributions that appear significantly similar to each other. The 4 or 5 kinds of probabilities that are indicated for every such quantitative comparison are concerned, respectively, with a few different standard assumptions about the nature of the two distributions being compared with each other, as follows: i) Student's $t$-test for checking the difference of the mean, when the distributions are thought to have the same variance; ii) the same when the two distributions are thought to have significantly different variance; iii) $F$-test for checking whether the two sample have a different variance; iv) $\chi^{2}$-test for checking whether the two distributions are different (such a test can be carried out only whenever the two distributions have the same number of elements); v) the same by means of the Kolmogorov-Smirnov test. The left column also contains: i) the (non-weighted) mean of $D$ including its standard deviation, plus ii) the weighted mean and its error bar. Sometimes some input datum was found having a formal vanishing standard deviation. In such a case, some small and arbitrary value $\left(10^{-12}\right)$ was assigned to it, by which the resulting weighted mean is almost completely determined by such small-error value(s), while its error bar results insignificantly small. In such a case, the standard deviation of the non-weighted mean can be used as an indicative error bar. 
AE sources are randomly 3D distributed in space. In contrast, when $D<1$ some organised evolution of the system is likely to be in progress, such as e.g., when the AE source distribution slowly evolves from 3D towards some comparatively more 2D space organisation.

\subsection{Comparing different tectonic settings}

Figure 3 and table II show the comparison between different settings. The formal statistical comparison is reported in detail between the distributions of $D$ concerned with the 6 different case histories. It is concluded that there are two sets of distributions that appear similar to each other, respectively: i) Vesuvius, Stromboli, and the laboratory sample, and ii) Gran Sasso and Potenza. Such two sets appear significantly different when they are compared with each other (table II). It is important to stress that the success of our analysis largely resulted from the possibility of comparing with each other analogous applications to physically different case histories, by which the actual heuristic potential of the AE diagnostic tool can be tested, and sensitivity and reliability assessed. Figure 3 and table I appear crucial in such respect.

According to the tentative intuitive rationale outlined in Section 3, Stromboli seems to represent a typical $\mathrm{AE}$ case history origi-

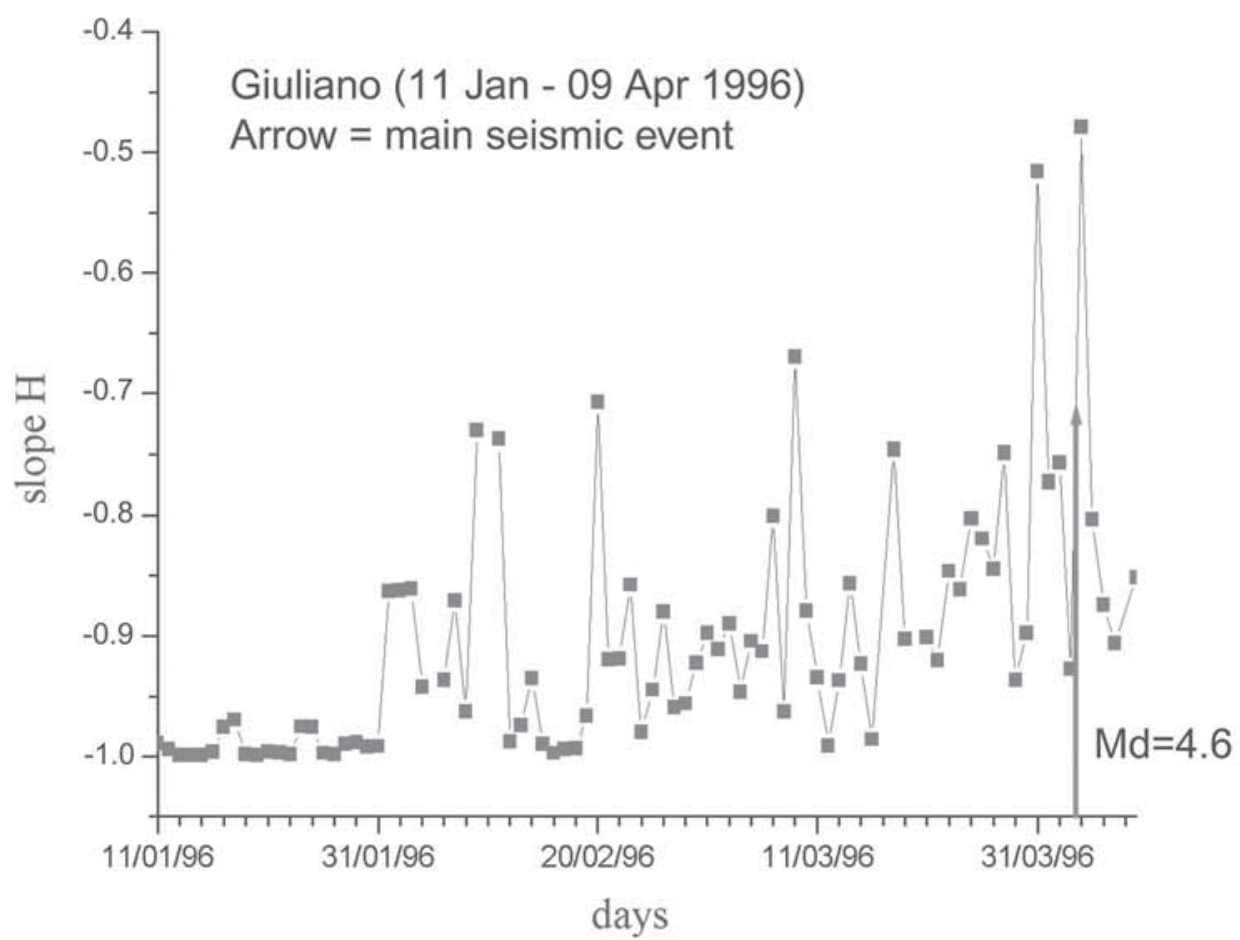

Fig. 4a. Time series of $H$ computed every day during a few months before the occurrence of the earthquake reported in fig. $4 \mathrm{~b}$, close to the recording station Giuliano. The system reveals a clear trend from random AE towards a progressively better organised phenomenon, characterised by rupture along some preferred plane, to be identified either with a fault plane, or maybe more likely (see text) with cleavage of some local solid structure. It is a complex system, originally disordered, which evolves toward an increasing order, until its mechanical structure yields. 
nated by phreatic diffusion. Vesuvius strongly resembles it. By this, any possibility that the late 1999 crisis of Vesuvius was originated by plutonic intrusions can be excluded. In addition, the laboratory sample simulates a dominant AE originated by pressure, with a lesser contribution by AE slip, shown by the lesser peak in its histogram in fig. 3 .

In contrast, the Giuliano site appears associated with an area where the faulting process generates some AE slip (either directly from the fault, or from a local structure, as per Section 3 ). Significantly smaller $D$ are detected. The same result is observed on Gran Sasso, where the AE is consequent to the thermal daily deformation of rocks, by which $\mathrm{AE}$ ought to be associated with slip $\mathrm{AE}$ occurring during cooling, i.e. by local nighttime (see Section 3).

All such conclusions are to be supported by suitable additional laboratory experiments (in progress) capable of significantly simulating several different pore-yielding conditions, and of testing the associated aforementioned tentative inferences.

\subsection{Time variation of fractal dimension}

Figure 4a shows the temporal variation of the tilt $H$ at Giuliano a few months before the occurrence of the earthquake reported in fig. $4 \mathrm{~b}$. Every point plotted in fig. $4 \mathrm{a}$ refers to the $\mathrm{AE}$

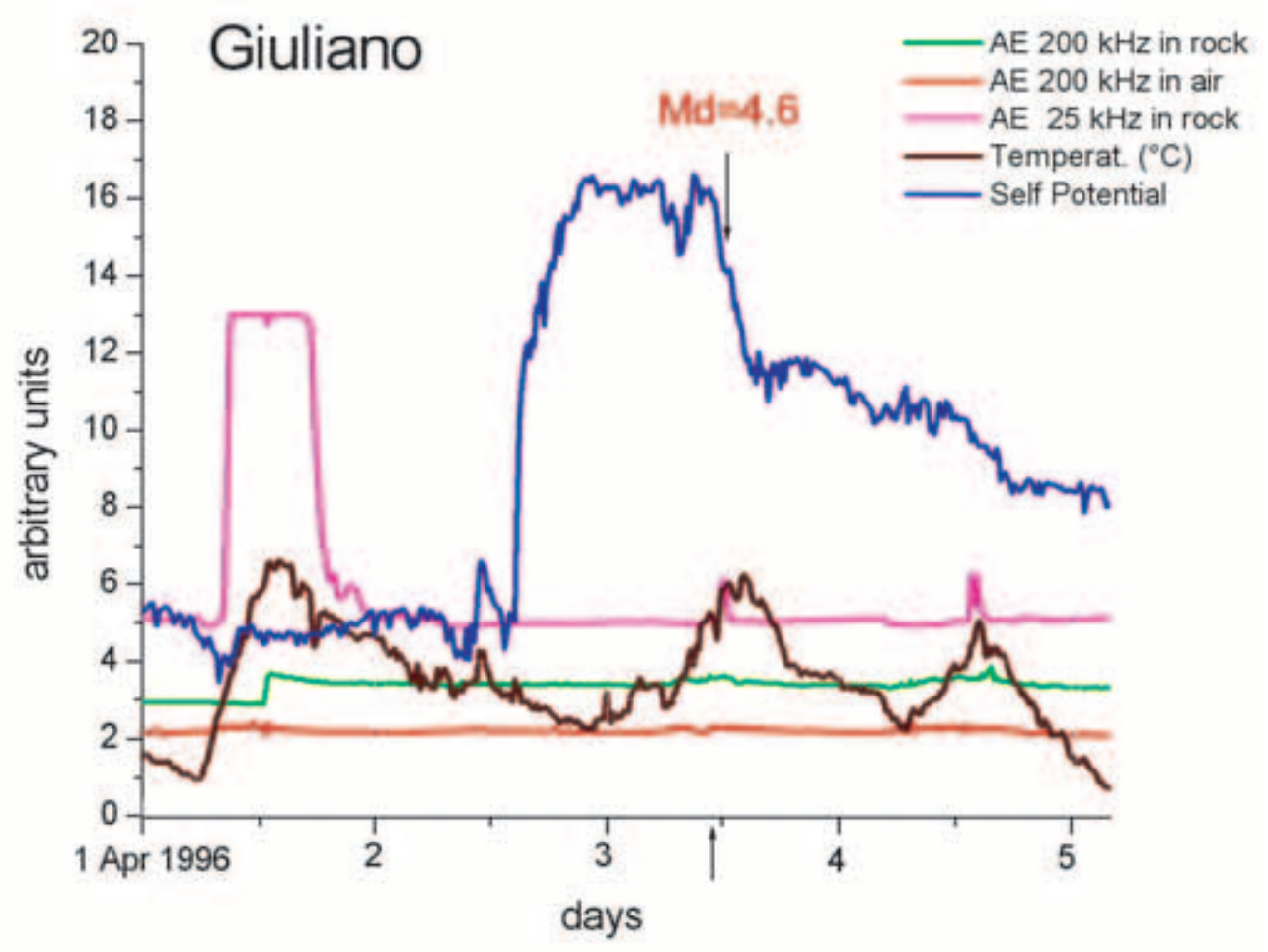

Fig. 4b. Time delay between the relative variations of $\mathrm{AE}$ at $25 \mathrm{kHz}$ (purple), the same at $200 \mathrm{kHz}$ in rock (green), and in air (red), plus self-potential (blue). Units on ordinate scale are arbitrary (see fig. 1). A $~ 2$ days precursor appears in the LF AE, and a lesser one in the HF AE in rock. This is associated with an increased porosity of ground, by which the fluid-flow increases, and one day later also the self-potential. When the self-potential starts recovering, an $M_{d}=4.6$ earthquake occurs with epicentre at $\sim 18.2 \mathrm{~km}$ from the recording site. Redrawn after Cuomo et al. (2000). 


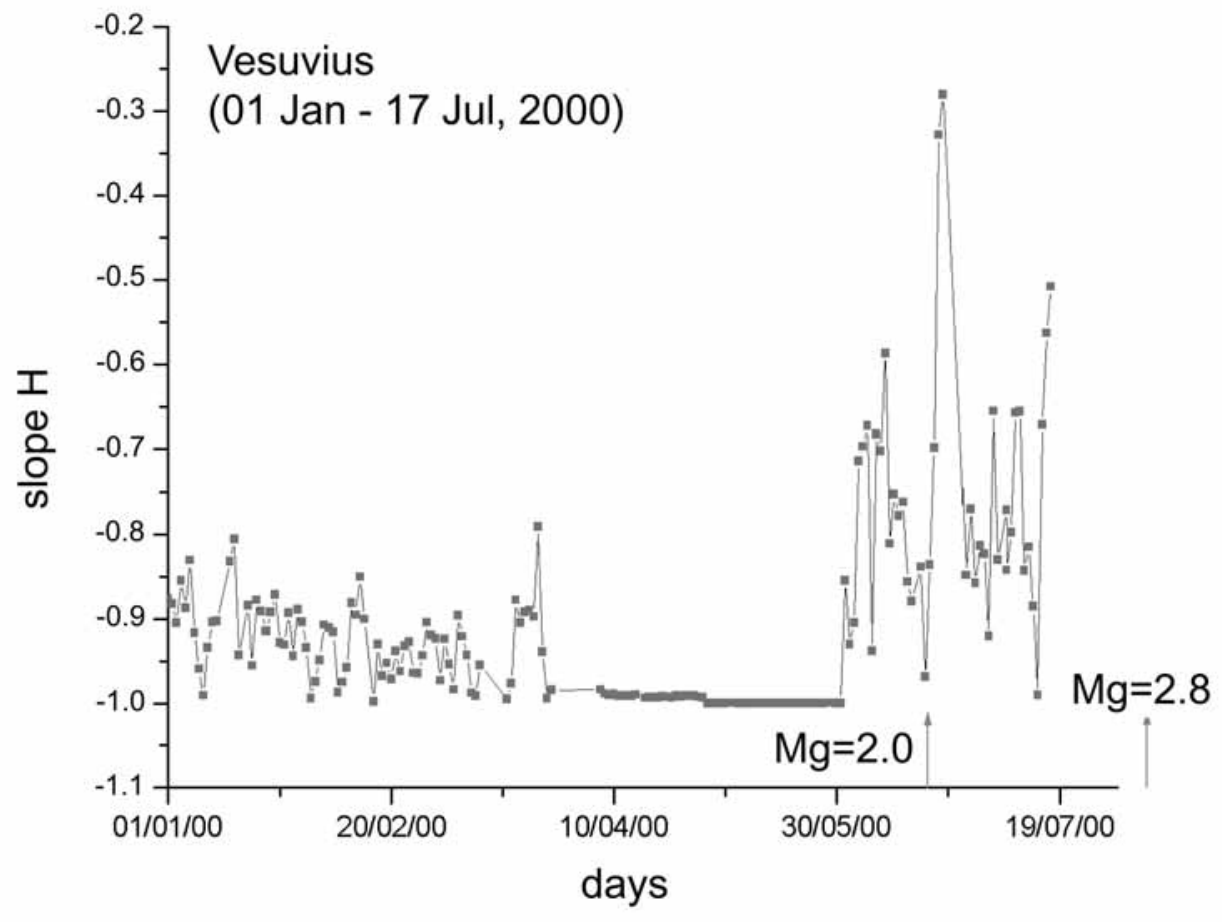

Fig. 5. Daily estimates of $H$ versus time of the AE collected on Vesuvius during January through June 2000 A.D. See text.

recorded during 1 day. The system appears progressively becoming more $2 \mathrm{D}$, i.e. towards some slip AE compared to a starting random AE. During earlier times only records of lesser accuracy were available. Concerning subsequent times, AE records are currently being analysed. In either case, there is clear evidence that it is always $H \sim-1$.

Figure 5 shows the temporal trend observed for Vesuvius during the first half of 2000 A.D. When $D \sim 1$. Vesuvius is during a charging phase, i.e. some hot fluid pressure, increasing versus time, diffuses into the pores producing a random $\mathrm{AE}$ and the system is thus «inflated». In contrast, when $D<1$. the endogenous fluid pressure is temporarily lowered, Vesuvius «deflates», the entire system collapses, much like a dome that is no longer supported from inside. Hence, the system eventually breaks along preferred crack or minifault planes (maybe, even almost microscopic) normally undetectable by standard observations. Such guess is supported by seismic activity. More shocks occur during inflation than during deflation. Deflation shocks are comparatively much stronger than inflation shocks. Consider the total energy integrated versus time during an entire period of inflation: it ought to be roughly the same when integrated during the subsequent deflation period. The eventual observed difference ought to reflect the seismic energy associated with shocks that are not detected by the seismic network, due either to instrument sensitivity, or to the damping of feeble signals within the ground (in progress). Better multiparametric records are recommended. In any case, the AE technique seems to provide the very first effective method for 
monitoring inflation/deflation of a volcano. The present analysis has a time resolution of \pm 1 day, although in principle the same method can be applied with a much higher detail (in progress).

\section{Conclusions}

According to our inferred evidence, AE seems to be a heuristically effective tool for investigating ground deformation, microfracturing, and the time variation of its porosity. The apparently most reasonable interpretation is in terms of a strictly local effect, released by geologic structure of limited extension and of great rigidity (such as dykes, rocks, lava, etc.) that are stressed and strained following some large-area tectonic or volcanic event. AE appears to be potential and useful complementary evidence in addition to, and in eventual correlation with, seismic and geochemical information.

It appears possible to distinguish phenomena triggered by the diffusion of some fluid into rock pores, yielding a temporal sequence of $\mathrm{AE}$ events characterised by a slightly higher fractal dimension $D$ (or equivalently a slightly steeper slope $H$ in the Richardson plot), compared to the case of AE originated by some strain during either crystal cleavage or fault sliding. A few laboratory experiments seem to support such inferences. Additional ones ought to be performed.

In general, the ultimate advantage of $\mathrm{AE}$ compared to the standard approach in terms of seismic activity relies on its implicit direct capability of probing processes that occur on the atomic or molecular scale, much before (i.e. several up to a few tens hours, or even several months in advance) a comparatively much larger seismic energy release. No matter whether such approach is more or less useful for mitigating volcanic and seismic hazards, its investigation ought to be exploited to assess some observational inference that apparently cannot be investigated by any other kind of observations, and that therefore can open unprecedented diagnostic applications.

Figures $4 \mathrm{a}$ and 5 ought to be considered as a possible indication (respectively) of an unprecedented know-how for monitoring areas where an earthquake is going to be prepared, and of an unprecedented possibility of monitoring «inflation» and «deflation» times of a volcano. It should be stressed, however, that it is possible to monitor the temporal evolution of a system for assessing under what circumstances an eventual hazard is going to evolve towards a risk, although simply by this no alert can be issued.

The next steps shall be twofold. On one side, an attempt will be made to improve the temporal resolution while monitoring the «inflation» and «deflation» times of a volcano. A second step shall attempt to recognise the chain-reaction that occurs during the time sequence of the yielding bonds of one single occurrence. The definition of «event» however shall be critical. The concept of microevent (lasting typically $\sim 10 \mathrm{~ms}$ ) still remains the same, being the elementary monitored element. However, some suitable total duration $\Delta t$ for defining the concept of «event» shall be chosen, eventually different from $\sim 25 \mathrm{~s}$ as in Section 2. The ultimate target is to search for a substantial improvement of the temporal resolution of AE monitoring, aimed at measuring additional and unprecedented degrees of freedom of the system, focusing as far as possible on its prime process, even on the atomic or molecular scale.

\section{REFERENCES}

Chiappini, M., G.P. Gregori, G. Paparo, C. Bellecci, G.M. Crisci, G. DE Natale, P. Favali, I. Marson, A. MELONI, B. ZOLESI and E. BosCHI (2002): Stromboli. A natural laboratory of environmental science, J. Volcan. Geotherm. Res., 113, 429-442.

Cox, K.G. (1989): The role of mantle plumes in the development of continental drainage patterns, Nature (London), 342, 873-877.

CRISCI, G.M., R. DE RosA, S. EsPERANÇA, R. MAZZUOLI and M. SonNino (1991): Temporal evolution of a three component system: the island of Lipari (Aeolian arc, Southern Italy), Bull. Volcanol., 53, 207-221.

Cuomo, V., V. Lapenna, M. Macchiato, I. Marson, G. PAPARO, D. PATElla and S. Piscitelli (2000): Geoelectrical and seismoacoustic anomalous signals jointly recorded close to an active fault system in Southern Apennines (Italy), Phys. Chem. Earth, 25 (3), 255-261.

Diodati, P., S. PiazZA, A. Del Sole and L. MasciovecChio (2001): Daily annual electromagnetic noise variation and acoustic emission on the Gran Sasso mountain, Earth Planet. Sci. Lett., 184, 719-724. 
EBERHART, M.E. (1999): Why things break, Sci. Am., 281 (4), 44-51.

ESPERANÇA, S. and G.M. CRISCI (1995): The Island of Pantelleria: a case for the development of DMM-HIMU isotopic composition in a long-lived extensional setting, Earth Planet. Sci. Lett., 136, 167-182.

ESPERANÇA, S., G.M. CRISCI, R. DE RosA and R. MAZZUOLI (1992): The role of the crust in the magmatic evolution of the island of Lipari (Aeolian islands, Italy), Contrib. Mineral. Petrol., 112, 450-462.

Giovannelli, F. (Editor) (2001): The bridge between the Big Bang and biology. Stars, planetary systems, atmospheres, volcanoes: their link to life, in Proceedings of a Meeting hold at Stromboli (Italy) on September 13-17, 1999, CNR, Roma, pp. 440.

Gregori, G.P. (1993): The next eruption of SommaVesuvius, in Schröder (1993), 191-213.

Gregori, G.P. (1996): The next eruption of SommaVesuvius, in Piccione and Antonelli (1996), 399-468.

GREGORI, G.P. (1998): Natural catastrophes and point-like processes. Data handling and prevision, Ann. Geofis., 41 (5/6), 767-786.

Gregori, G.P. and WenJIE DONG (1996): The correlation between the geomagnetic field reversals, the Hawaiian vulcanism and the motion of the Pacific plate, Ann. Geofis., 39 (1), 49-65.

Gregori, G.P., V.P. BAnzon, R. LeOnARdi and G. DE FRANCESCHI (1992): Geomagnetic activity versus volcanic cycles, and their forecasting. Application to Etna and Vesuvius, in Schröder and Legrand (1992), 188-222.

GREGORI, G.P., V. BANZON and R. LEONARDI (1994): The cycles of volcanoes, and the global synchronism of the time variation of their heat source, in Schröder and Colacino (1994), 152-191.

Gregori, G.P., C. Bellecci, C. Bianchi, G.M. Crisci, G. DE Franceschi, G. Di Stefano, G. Etiope, E.S. KAZIMIROVSKY, I. MARSON, G. PAPARO, E. PIERVITALI, G. Romeo, C. ScotTo, Q. TACCETTI and B. Zolesi (2001): The global atmospheric-electrical circuit, mesospheric electrical phenomena, particle precipitation, volcanoes like lightning rods, and the mechanisms of climate, Presented at the IUGG General Assembly, Birmingham (U.K.), July 1999.

Mogi, K. (1985): Earthquake Prediction, Academic Press (Harcourt Brace Jovanovich, Publ.), Tokyo etc., pp. 355.

NuR, A. (1972): Dilatancy pore fluids, and premonitory variations of $t_{p} / t_{s}$ travel times, Bull. Seismol. Soc. Am., 62, 1217-1222

PAPARO, G. and G.P. GREGORI (2001): Volcanoes and environment and the natural laboratory of Stromboli, in Giovannelli (2001), 289-309.

Petri, A., G. Paparo, A. Vespignani, A. Alippi and M. COSTANTINI (1994): Experimental evidence for critical dynamics in microfracturing processes, Phys. Rev. Lett., 73 (25), 3423-3426.

Piccione, V. and C. Antonelli (Editors) (1996): Proceedings of the 4th Workshop of Progetto Strategico Clima, Ambiente e Territorio nel Mezzogiorno, Lecce, November 11-14, 1991, 2 vol., pp. 644, CNR, Roma.

RIKITAKE, T. (1976): Earthquake Prediction (Elsevier, Amsterdam etc.), pp. 357.

SCHRÖDER, W. (Editor) (1993): The Earth and the universe, Festschrift in honour of Prof. H.-J. Treder, Newsletter of IDCH-IAGA, (20), 1-497, Science Edition, BremenRoennebeck.

SCHRÖDER, W. and J.P. LEGRAND (Editors) (1992): Solar Terrestrial Variability and Global Change, pp. 243, IDCH of IAGA, Bremen-Roennebeck.

SCHRÖDER, W. and M. COLACINO (Editors) (1994): Geophysics: past achievements and future challenges, Newsletter of IDCH-IAGA, (20), 1-191, Science Edition / IDCH of IAGA, Bremen-Roennebeck.

ScotT, I.G. (1991): Basic Acoustic Emission (Gordon and Breach Science Publishers, New York, etc.), pp. 246.

TURCOTTE, D.L. (1992): Fractals and Chaos in Geology and Geophysics (Cambridge Univ. Press), pp. 221.

Vespignani, A., A. Petri, A. Alippi, G. PAPARo and M. COSTANTINI (1995): Long range correlation properties of aftershock relaxation signals. Fractals, 3 (4), 839-847. 